\title{
Spatial Jitter Influence on the Average BLER Performance of SIMO FSO Links over Atmospheric Turbulence Channels
}

\author{
Nikolaos A. Androutsos, Hector E. Nistazakis *D, Efstratios V. Chatzikontis, Argyris N. Stassinakis \\ and George S. Tombras
}

Citation: Androutsos, N.A.; Nistazakis, H.E.; Chatzikontis, E.V.; Stassinakis, A.N.; Tombras, G.S. Spatial Jitter Influence on the Average BLER Performance of SIMO FSO

Links over Atmospheric Turbulence Channels. Electronics 2021, 10, 2033. https://doi.org/10.3390/ electronics10162033

\section{Academic Editors:}

Hirokazu Kobayashi and Imran Shafique Ansari

Received: 4 July 2021

Accepted: 19 August 2021

Published: 22 August 2021

Publisher's Note: MDPI stays neutral with regard to jurisdictional claims in published maps and institutional affiliations.

Copyright: (C) 2021 by the authors Licensee MDPI, Basel, Switzerland. This article is an open access article distributed under the terms and conditions of the Creative Commons Attribution (CC BY) license (https:/ / creativecommons.org/licenses/by/ $4.0 /)$.
Section of Electronic Physics and Systems, Department of Physics, National and Kapodistrian University of Athens, 15784 Athens, Greece; nickandr@phys.uoa.gr (N.A.A.); stratisch@phys.uoa.gr (E.V.C.); a-stasinakis@phys.uoa.gr (A.N.S.); gtombras@phys.uoa.gr (G.S.T.)

* Correspondence: enistaz@phys.uoa.gr; Tel.: +30-210-7276-710
Abstract: In the recent years, Free Space Optics (FSO) technology has attracted significant research and commercial interest mostly because of its many advantages in comparison with other radio systems used for point-to-point connections. However, the reliable operation of these systems significantly depends on the conditions of the atmosphere in the area in which the optical beam propagates. The most important of these conditions are atmospheric turbulence and the misalignment between the optical beam and the receiver, which is also known as the pointing errors effect. In this work, in order to obviate the performance mitigation caused by these phenomena, we examined the most widely accepted and one of the most effective techniques, i.e., the implementation of receivers' diversity. Various metrics have been investigated to evaluate the performance of such systems, but most of them do not take into account that the ultra-fast modern optical communication systems use blocks of bits for the transmission and codes for the detection and/or correction of erroneous bits. Thus, by taking these aspects into account, in this work, we investigated the combined impact of spatial jitter and atmospheric turbulence on the total average block error rate of an optical wireless system with receivers' diversity. Novel closed-form analytical formulas were derived.

Keywords: FSO; SIMO; ABLER; turbulence; pointing errors; spatial jitter; diversity

\section{Introduction}

Over the last few years, FSO technology has raised significant scientific interest for experimental, theoretical, and commercial purposes, mostly because of its many advantages in comparison with other techniques such as those based on Radio Frequency (RF) systems for point-to-point (P2P) or point-to-multipoint applications. The most important advantages are the very high capacity, the permissible high bandwidth, the operation in a band of the electromagnetic spectrum for which there is no required license, high safety, along with very low operation and installation cost and the low power consumption [1-4]. FSO systems are a promising solution to complement conventional radio frequency systems or fiber optics [5] in cases where point-to-point connections can be used.

However, as expected for every wireless communication system, the operation, performance, and reliability of FSO systems strongly depend on the atmospheric conditions and other phenomena that prevail in the area where the optical beam propagates [1]. The most severe of these phenomena are atmospheric turbulence, which is an outcome of the variation of the refractive index of the atmosphere [6], and the misalignment between the optical beam and the reception node, which is mainly the result of building sways [6] in which the FSO equipment is usually located and is known as the pointing errors effect. Atmospheric turbulence causes rapid irradiance fluctuations on the received signal as a consequence of the variations in the refractive index, because of inhomogeneities in pressure and temperature [1], which is the so-called scintillation effect [4,7-13]. On the other hand, the vibrations of the transmitter impose vibrations on the optical beam, and 
the result is a wrong alignment between the optical beam and the receiver, i.e., the pointing errors effect $[1,6,10,14,15]$. Hence, the combined impact of these severe phenomena may have an extremely damaging effect on the reliability and performance of the FSO system. In order to overcome such limitations due to a turbulent atmosphere and a wrong alignment, several methods and techniques have been presented and studied in the literature. In some cases, in order to obviate these negative effects, it is feasible to simply increase the transmission power, but for large propagation distances, safety regulations of laser operations and other technical limitations set restrictions on power increment to reach the required level [16]. Thus, one of the most effective and widely accepted, is the deployment of diversity in the receivers, which is very popular in wireless RF systems, [16], and can be used also in FSO. The implementation of diversity mentioned to the transmission of many copies of the same signal from a transmitter towards one or multiple receivers, so as to be maintained the required performance level of the FSO during a poor propagation of the laser beam through the atmosphere, [1]; this configuration works as a single-input multipleoutput (SIMO) scheme. This technique may be generally implemented in terms of space, time, or wavelength [1,16-18]. In the case of spatial diversity [17,18], the sending node transmits multiple copies of the initial signal towards many receivers which are located in different places, while in the case of time diversity, there is a single receiver, but each signal is emitted many times in different timeslots [1,16]. Finally, in the case of wavelength diversity, the signal is transmitted towards multiple receivers but in different propagation wavelengths $[1,16]$. It is expected that, the implementation of spatial and wavelength diversity regimes can increase significantly the cost of the systems but can also enhance significantly their reliability, given the fact that the information is propagated through different physical paths or wavelengths. On the other hand, the implementation of the time diversity regime is easier and has a lower cost, as only a pair of transmitter-receiver has to be installed, but may results in time delays, data rate reductions, and lower reliability than the other regimes, given the fact that it uses only a physical path with only a single pair of transmitter-receiver, [16].

For the performance evaluation of such links, several metrics have been proposed in the literature, but most of them do not take into account that the ultra-fast modern optical communication systems use blocks of bits for the transmission and codes for the detection and/or correction of erroneous bits. An effective metric for the accurate evaluation of the reliability of optical wireless links and for choosing the appropriate coding method for each individual FSO system is the average Block Error Rate (ABLER), which corresponds to the probability that more than $M$ error bits occur in a specific block of $N$ bits, $[14,19]$.

For our study, we assumed a single-input multiple-output (SIMO) FSO system and implemented receivers' diversity with spatial or wavelength realizations. In both spatial and wavelength diversity scenarios, we considered that the sending node transmitted simultaneously $D$ copies of the same part of the information signal towards $D$ receivers, which were either located in different places or operating in different wavelengths. In both cases, the same initial signal propagated through paths with alternative physical characteristics, and the total signal consisted of $D$ different alternative distorted signals [1]. Moreover, the intensity modulation/direct detection (IM/DD) method with L-symbols pulse position modulation (L-PPM) and $L$-symbols pulse amplitude modulation (L-PAM) and the optimal combining (OC) method for signal reception were considered. It should be mentioned here that L-PPM and L-PAM along with On-Off Keying (OOK) modulation schemes are the most widely accepted for FSO systems. However, in this work, we chose the PPM and PAM schemes, which are mathematically more complex but in marginal cases, include other modulation formats. To describe scintillation, we considered the GammaGamma statistical distribution, which is appropriate for a weak to strong turbulence, while to determine pointing errors influence, we used the well-known Rayleigh distribution for the modeling of the radial displacement at the receiver $[6,10]$.

Thus, by taking all the aforementioned into account, in this study, we examined the reliability of a SIMO FSO system operating over composite channels with turbulence and 
pointing errors, in terms of ABLER metrics and we derived the corresponding closed-form and novel analytical expressions. It should be mentioned here that, to the best of our knowledge at this time, there are no other works investigating the ABLER performance of a SIMO FSO system over such composite channels with scintillation and spatial jitter effect.

The remainder of this work is organized as follows: in Section 2, we introduce the system under investigation and the model of the channel considering the joint influence of scintillation and spatial jitter, while in Section 3, the total ABLER of the SIMO FSO system is investigated. Next, in Section 4, we present the corresponding numerical results, while the concluding remarks are presented in Section 5.

\section{System and Channel Model}

The statistical channel model can be given as $[1,3,16]$ :

$$
s_{d}=\eta_{d} x I_{d}+n, \text { with } d=1,2, \ldots, D,
$$

where $s_{d}$ represents each one of the $D$ optical signals at the corresponding receiver, $\eta_{d}$ stands for the effective photocurrent conversion ratio in each receiver, $I_{d}$ represents the normalized received irradiance of the optical signal of each of the $D$ receivers, $x$ stands for the modulated signal which can take the well-known binary values " 1 " and " 0 ", and $n$ is the additive white Gaussian noise (AWGN) with zero mean and variance equal to $N_{0} / 2[1,3]$. The total field of view (FOV) of all detectors, in practical systems with multiple photodetectors, has to be covered by the optical beam footprint in the receivers' telescope [16]. This implies that the area of each detector is $D$ times less, compared with the area without diversity [16]. Thus, the variance of the noise in each photodetector will be $D$ times less in comparison with the noise variance of a system where there is no diversity, [16].

The total received irradiance is expressed as $[10,20,21]$ :

$$
I_{d}=I_{t, d} I_{p, d} I_{l, d},
$$

where $I_{t, d}$ and $I_{p, d}$ are the values of irradiance due to scintillation and spatial jitter for each one of the $D$ receivers, respectively, and $I_{l, d}$ represents the parameter of path losses which is deterministic and, without loss of generality, has been considered normalized to unity $[1,3]$.

\subsection{Turbulence Model}

In order to describe turbulence-induced irradiance fluctuations, we considered the Gamma-Gamma distribution, which is appropriate for weak to strong turbulence. Its probability density function (PDF) is given as [3,22,23]:

$$
f_{I_{t, d}}\left(I_{t, d}\right)=\frac{2\left(\alpha_{d} \beta_{d}\right)^{\frac{\alpha_{d}+\beta_{d}}{2}}}{\Gamma\left(\alpha_{d}\right) \Gamma\left(\beta_{d}\right)} I_{t, d}^{\alpha_{d}+\beta_{d}}-1 K_{\alpha_{d}-\beta_{d}}\left(2 \sqrt{\alpha_{d} \beta_{d} I_{t, d}}\right),
$$

where $K_{v}($.$) represents the modified Bessel function of the second kind of order v$, expression (8.432.2) of Ref. [24], and $\Gamma($.$) is the Gamma function, expression (8.310.1) of Ref. [24]. The$ parameters $\alpha_{d}$ and $\beta_{d}$ depend on the values of other link's characteristics such as the refractive index structure parameter, the link's length, the receiver's aperture diameter, and the operational wavelength and can be estimated as described $[3,22,25,26]$.

\subsection{Pointing Errors Model}

For the pointing errors impact, the independent identical Gaussian distributions for the elevation and the horizontal displacement were taken into account, while a Rayleigh 
distribution was assumed for the receiver's radial displacement [15]. Thus, the PDF of $I_{p, d}$ was obtain as $[15,27,28]$ :

$$
f_{I_{p, d}}\left(I_{p, d}\right)=\frac{\psi_{d}^{2}}{A_{0, d}^{\psi_{d}^{2}}} I_{p, d}^{\psi_{d}^{2}-1}, 0 \leq I_{p, d} \leq A_{0, d},
$$

where $\psi_{d}=w_{z_{e q, d}} / 2 \sigma_{s, d}$ is the fraction of the equivalent beam radius in the receiver and the pointing errors displacement standard deviation at each receiver [15]. Additionally, $w_{z, e q, d}^{2}=\sqrt{\pi} e r f\left(u_{d}\right) w_{z, d}^{2} /\left(2 u_{d} e^{-u_{d}^{2}}\right), u_{d}=\sqrt{\pi} r_{d} / \sqrt{2} w_{z, d}$, while $A_{0, d}=\left[\operatorname{erf}\left(u_{d}\right)\right]^{2}$, with $\operatorname{erf}(\cdot)$ being the error function from equation (8.250.1) of Ref. [24]. Moreover, $r_{d}$ stands for the aperture radius of the receiver, while $w_{z, d}$ represents the Gaussian beam's waist at a $z_{d}$ propagated distance from the transmitter, [1].

\subsection{Composite Irradiance Model}

By considering composite channels with scintillation-induced fading and misalignment induced fading, the PDF of the normalized received irradiance $I_{d}$ is obtained through the integral $[3,16,22]$ :

$$
f_{I_{d}}\left(I_{d}\right)=\int f_{I_{d} \mid I_{t, d}}\left(I_{d} \mid I_{t, d}\right) f_{I_{t, d}}\left(I_{t, d}\right) d I_{t, d}
$$

where $f_{I_{d} \mid I_{t, d}}\left(I_{d} \mid I_{t, d}\right)$ is the conditional probability of the irradiance $I_{t, d}$. Then, by appropriately substituting expressions (3) and (4) into the integral of (5), the composite model with Gamma-Gamma modeled turbulence and misalignment is obtained [22]:

$$
f_{I_{d}}\left(I_{d}\right)=\frac{\alpha_{d} \beta_{d} \psi_{d}^{2}}{A_{0, d} \Gamma\left(\alpha_{d}\right) \Gamma\left(\beta_{d}\right)} G_{1,3}^{3,0}\left(\frac{\alpha_{d} \beta_{d} I_{d}}{A_{0, d} g_{d}} \mid \begin{array}{c}
\psi_{d}^{2} \\
\psi_{d}^{2}-1, \alpha_{d}-1, \beta_{d}-1
\end{array}\right),
$$

where G[.] denotes the Meijer function, Equation (5) of Ref. [29]. Additionally, the instantaneous and the expected signal-to-noise ratios (SNRs) of each branch of the SIMO system can be given as $\gamma_{d}=\left(\eta_{d} I_{d}\right)^{2} / N_{0}$ and $\bar{\gamma}_{d}=\left(\eta_{d} E\left[I_{d}\right]\right)^{2} / N_{0}$, respectively [1], where $E\left[I_{d}\right]$ represents the corresponding expected normalized irradiance value, which can be obtain by substituting expression (6) into the integral $E\left[I_{d}\right]=\int_{0}^{\infty} I_{d} f\left(I_{d}\right) d I_{d}$ and, according to [3], is given as:

$$
E\left[I_{d}\right]=A_{0, d}\left(1+\psi_{d}^{-2}\right)^{-1}
$$

\section{Average BLER Estimation}

BLER performance represents the probability that more than $M$-bit error detections occur in a block composed of $\mathrm{N}$-bits [14] and is a significant and realistic metric for the to accurate estimate of the reliability of FSO links. To characterize a block of $N$ bits as faulty, more than $M$ error bits in this block have to be detected. Thus, BLER expression which represents this probability is $[14,19,30]$ :

$$
P\left(M, N ; I_{d}\right)=\sum_{m=M+1}^{N}\left(\begin{array}{l}
N \\
m
\end{array}\right) p^{m}(1-p)^{N-m},
$$

where $p$ denotes the probability to receive an error bit. Thus, for our scenario with receivers diversity and by using the OC method, the probability $p$ in the case of $L-P P M$ modulation is [3]:

$$
p_{L-P P M}=\frac{L}{2} Q\left(\sqrt{L \log _{2}(L) \sum_{d=1}^{D} \frac{\left(\eta_{d} I_{d}\right)^{2}}{4 D N_{0}}}\right),
$$


While, for L-PAM, it is obtained after appropriate modifications of the corresponding expression of [3], as:

$$
p_{L-P A M}=\frac{2(L-1)}{L \log _{2}(L)} Q\left(\sqrt{\frac{\log _{2}(L)}{(L-1)^{2}} \sum_{d=1}^{D} \frac{\left(\eta_{d} I_{d}\right)^{2}}{2 D N_{0}}}\right),
$$

where $Q($.) represents the well-known $Q$-function. By averaging the BLER expression of (8), the average $B L E R$ expression is obtain [14,19]:

$$
A B L E R=\int_{\vec{I}} P(M, N ; \vec{I}) f_{\vec{I}}(\vec{I}) d \vec{I},
$$

where $\vec{I}=\left(I_{1}, I_{d} \ldots I_{D}\right)$ represents the vector of the signal with $D$ components, arriving at the receivers of the system [1]. We introduced expression (8) into the integral of expression (11), along with the application of the binomial expansion formula in the term $(1-p)^{N-m}$. Then, the product $p^{m}(1-p)^{N-m}$ takes the form $\sum_{k=0}^{N-m}\left(\begin{array}{c}N-m \\ k\end{array}\right)(-1)^{k} p^{m+k}$, $[14,19]$, and the ABLER expression was obtained as:

$$
A B L E R=\int_{\vec{I}} \sum_{m=M+1}^{N}\left(\begin{array}{c}
N \\
m
\end{array}\right) \sum_{k=0}^{N-m}\left(\begin{array}{c}
N-m \\
k
\end{array}\right)(-1)^{k} p^{m+k} f_{\vec{I}}(\vec{I}) d \vec{I}
$$

where $p$ can be given by either expression (9) or expression (10). Next, by replacing the $Q$-function into expressions (9) and (10), with the approximation $Q(x) \approx 0.208 e^{-0.971 x^{2}}+0.147 e^{-0.525 x^{2}}$ of Ref. [31], along with the application of the multinomial formula for the exponential terms, the $A B L E R$ expression in the case of $L-P P M$ modulation is:

$$
\begin{gathered}
A B L E R_{L-P P M} \approx \int_{\vec{I}} \sum_{m=M+1}^{N}\left(\begin{array}{c}
N \\
m
\end{array}\right) \sum_{k=0}^{N-m}\left(\begin{array}{c}
N-m \\
k
\end{array}\right)(-1)^{k}\left(\frac{L}{2}\right)^{m+k} \times \\
\times \sum_{t=0}^{m+k}\left(\begin{array}{c}
m+k \\
t
\end{array}\right)\left(0.208 \prod_{d=1}^{D} e^{-0.971 L \log _{2}(L) \frac{\left(\eta_{d} I_{d}\right)^{2}}{4 D N_{0}}}\right)^{m+k-t} \times \\
\times\left(0.147 \prod_{d=1}^{D} e^{-0.525 L \log _{2}(L) \frac{\left(\eta_{d} I_{d}\right)^{2}}{4 D N_{0}}}\right)^{t} f_{\vec{I}}(\vec{I}) d \vec{I}
\end{gathered}
$$

while, for $L-P A M$ it is derived as:

$$
\begin{aligned}
& A B L E R_{L-P P M} \approx \int_{I} \sum_{m=M+1}^{N}\left(\begin{array}{c}
N \\
m
\end{array}\right) \sum_{k=0}^{N-m}\left(\begin{array}{c}
N-m \\
k
\end{array}\right)(-1)^{k}\left(\frac{2(L-1)}{L \log _{2}(L)}\right)^{m+k} \times \\
& \times \sum_{t=0}^{m+k}\left(\begin{array}{c}
m+k \\
t
\end{array}\right)\left(0.208 \prod_{d=1}^{D} e^{\frac{-0.971 \log _{2}(L)}{(L-1)^{2}} \times \frac{\left(\eta_{d} I_{d}\right)^{2}}{2 N_{0}}}\right)^{m+k-t} \times \\
& \times\left(0.147 \prod_{d=1}^{D} e^{\frac{-0.525 \log _{2}(L)}{(L-1)^{2}} \times \frac{\left(\eta_{d} I_{d}\right)^{2}}{2 D N_{0}}}\right)^{t} f_{\vec{I}}(\vec{I}) d \vec{I}
\end{aligned}
$$


Then, by substituting the composite PDF of Equation (6) into the above expressions (13) and (14), we obtained for L-PPM:

$$
\begin{aligned}
& A B L E R_{L-P P M} \approx \prod_{d=1}^{D}\left[\sum_{m=M+1}^{N} \sum_{k=0}^{N-m} \sum_{t=0}^{m+k}\left(\begin{array}{l}
N \\
m
\end{array}\right)\left(\begin{array}{c}
N-m \\
k
\end{array}\right) \times\right. \\
& \times\left(\begin{array}{c}
m+k \\
t
\end{array}\right) \frac{(-1)^{k} \alpha_{d} \beta_{d} \psi_{d}^{2}}{A_{0, d} g_{d} \Gamma\left(\alpha_{d}\right) \Gamma\left(\beta_{d}\right)}\left(\frac{L}{2}\right)^{m+k} \times 0.208^{m+k-t} \times 0.147^{t} \times \\
& \left.\int_{0}^{\infty} e^{x I_{d}^{2}} G_{1,3}^{3,0}\left(\frac{\alpha_{d} \beta_{d}}{A_{0, d} g_{d}} I_{d} \mid \psi_{d}^{2}-1, \alpha_{d}-1, \beta_{d}^{2}-1\right) d I_{d}\right],
\end{aligned}
$$

and for L-PAM:

$$
\begin{aligned}
& A B L E R_{L-P A M} \approx \prod_{d=1}^{D}\left[\sum_{m=M+1}^{N} \sum_{k=0}^{N-m} \sum_{t=0}^{m+k}\left(\begin{array}{l}
N \\
m
\end{array}\right)\left(\begin{array}{c}
N-m \\
k
\end{array}\right) \times\right. \\
& \times\left(\begin{array}{c}
m+k \\
t
\end{array}\right) \frac{(-1)^{k} \alpha_{d} \beta_{d} \psi_{d}^{2}}{A_{0, d} g_{d} \Gamma\left(\alpha_{d}\right) \Gamma\left(\beta_{d}\right)}\left(\frac{2(L-1)}{L \log _{2}(L)}\right)^{m+k} \times 0.208^{m+k-t} \times 0.147^{t} \times \\
& \left.\int_{0}^{\infty} e^{y I_{d}^{2}} G_{1,3}^{3,0}\left(\frac{\alpha_{d} \beta_{d}}{A_{0, d} g_{d}} I_{d} \mid \begin{array}{c}
\psi_{d}^{2} \\
\psi_{d}^{2}-1, \alpha_{d}-1, \beta_{d}-1
\end{array}\right) d I_{d}\right],
\end{aligned}
$$

where

$$
\begin{aligned}
& x=\frac{L \log _{2}(L) \bar{\gamma}_{d}}{4 D E^{2}\left[I_{d}\right]}(-0.971 m-0.971 k+0.446 t), \\
& y=\frac{\log _{2}(L) \bar{\gamma}_{d}}{2(L-1)^{2} D E^{2}\left[I_{d}\right]}(-0.971 m-0.971 k+0.446 t) .
\end{aligned}
$$

Next, by using the transformation (01.03.26.0004.01) of Ref. [32], we represented the exponential terms through their corresponding term of Meijer-G function and, by implementing the integral transformation (07.34.21.0013.01) of Ref. [32], the closed-form analytical expression for the ABLER estimation of the FSO system with receivers' diversity operating over the combined influence of turbulence and pointing errors for the $L-P P M$ modulation case was derived as:

$$
\begin{gathered}
A B L E R_{L-P P M} \approx \prod_{d=1}^{D}\left[\sum_{m=M+1}^{N} \sum_{k=0}^{N-m} \sum_{t=0}^{m+k}\left(\begin{array}{c}
N \\
m
\end{array}\right)\left(\begin{array}{c}
N-m \\
k
\end{array}\right) \times\right. \\
\times\left(\begin{array}{c}
m+k \\
t
\end{array}\right) \frac{(-1)^{k} \times 2^{\alpha_{d}+\beta_{d}-2} \times 0.208^{m+k-t} \times 0.147^{t} \times \psi_{d}^{2}}{2 \pi \Gamma\left(\alpha_{d}\right) \Gamma\left(\beta_{d}\right)}\left(\frac{L}{2}\right)^{m+k} \times \\
\left.G_{6,3}^{1,6}\left(\frac{-16 x\left(A_{0, d} g_{d}\right)^{2}}{\left(\alpha_{d} \beta_{d}\right)^{2}} \mid \begin{array}{c}
\frac{1-\psi_{d}^{2}}{2}, \frac{2-\psi_{d}^{2}}{2}, \frac{1-\alpha_{d}}{2}, \frac{2-\alpha_{d}}{2}, \frac{1-\beta_{d}}{2}, \frac{2-\beta_{d}}{2} \\
0, \frac{-\psi_{d}^{2}}{2}, \frac{1-\psi_{d}^{2}}{2}
\end{array}\right)\right],
\end{gathered}
$$

while, for $L-P A M$ it was derived as:

$$
\begin{gathered}
A B L E R_{L-P A M} \approx \prod_{d=1}^{D}\left[\sum_{m=M+1}^{N} \sum_{k=0}^{N-m} \sum_{t=0}^{m+k}\left(\begin{array}{c}
N \\
m
\end{array}\right)\left(\begin{array}{c}
N-m \\
k
\end{array}\right) \times\right. \\
\times\left(\begin{array}{c}
m+k \\
t
\end{array}\right) \frac{(-1)^{k} \times 2^{\alpha_{d}+\beta_{d}-2} \times 0.208^{m+k-t} \times 0.147^{t} \times \psi_{d}^{2}}{2 \pi \Gamma\left(\alpha_{d}\right) \Gamma\left(\beta_{d}\right)}\left(\frac{2(L-1)}{L \log _{2}(L)}\right)^{m+k} \times \\
\left.G_{6,3}^{1,6}\left(\frac{-16 y\left(A_{0, d} d_{d}\right)^{2}}{\left(\alpha_{d} \beta_{d}\right)^{2}} \mid \begin{array}{c}
\left.\left.\frac{1-\psi_{d}^{2}}{2}, \frac{2-\psi_{d}^{2}}{2}, \frac{1-\alpha_{d}}{2}, \frac{2-\alpha_{d}}{2}, \frac{1-\beta_{d}}{2}, \frac{2-\beta_{d}}{2}\right)\right], \\
0, \frac{-\psi_{d}^{2}}{2}, \frac{1-\psi_{d}^{2}}{2}
\end{array}\right)\right]
\end{gathered}
$$

\section{Numerical Results}

In this part of our work, we present only some indicative numerical results of realistic scenarios derived from the ABLER estimation analytical expressions (18) and (19). It should be noticed here that the above-mentioned equations can be used as tools for $A B L E R$ performance estimation for any parameter set, depending on the specific FSO link under consideration. Additionally, we examine the accuracy of the model for marginal cases, 
by presenting Monte Carlo (MC) simulations. The FSO system under investigation was a SIMO operating through a turbulent atmospheric channel along with pointing errors, using PPM or PAM modulation formats. It should be mentioned that, for reasons of simplicity and without loss of generality, for the realization of both types of diversity and for all $D$ different transmissions of the same signal, we considered here, in the indicative cases below, that all paths and branches of the SIMO system had the same characteristics and, thus, we assumed $\alpha_{1}=\alpha_{d}=\ldots \alpha_{D}=\alpha$ and $\beta_{1}=\beta_{d}=\ldots \beta_{D}=\beta$ for the parameters of Gamma-Gamma distribution. Furthermore, we also considered the same assumptions for the pointing error parameters $\psi_{1}=\psi_{d}=\ldots \psi_{D}=\psi$ and consequently for the expected SNRs $\bar{\gamma}_{1}=\bar{\gamma}_{d}=\ldots \bar{\gamma}_{D}=\bar{\gamma}$. More precisely, for the turbulence parameters, we assumed two sets of parameters, as in previous works [33]. The first one $\alpha=1, \beta=2$, was considered to correspond to strong turbulence conditions, while the second one, $\alpha=2, \beta=5$, corresponded to weaker turbulence conditions. For the pointing errors impact, we also investigated two indicative scenarios. The first one, with $\left(\sigma_{s} / r, w_{z} / r\right)=(4,16)$, led to $\psi=2$, and the second one, where $\left(\sigma_{s} / r, w_{z} / r\right)=(4,24)$, led to $\psi=3$, corresponding to weaker pointing errors effect. For the investigation of diversity, we considered cases with $D=1,2$, or 3 different transmissions with more than $M=3$ or 4 erroneous bits, in a block of $N=5$ bits, assuming $L=4$ symbols for PPM and $L=8$ symbols for PAM modulation formats. However, any other set of parameters $N$ and $M$ for the ABLER metric and $L$ for the symbols of the modulation format could be selected, depending on the design of each FSO link.

In order to verify the accuracy of our model, we tried to proceed with the corresponding MC simulations. However, due to the form of the above-extracted expressions (15) and (16), the MC procedure could not be applied. Thus, we chose a marginal case for the ABLER estimation with PPM, $L=4, N=4, M=1$, Gamma-Gamma parameters' values $\alpha=1$ and $\beta=2$, for practically negligible pointing errors and we provide further realistic numerical results, accompanied by the corresponding MC simulations, by using $10^{6}$ samples.

In Figures 1-4, the performance of the SIMO FSO system over the combined impact of turbulence and pointing errors is presented in terms of ABLER versus the expected SNR. As shown in Figures 1-4, as larger the value of $D$, the performance of the system shows significant improvement. Moreover, the scenario with $\alpha=2, \beta=5$ and $\psi=3$ outperformed the one with $\alpha=1, \beta=2$ and $\psi=2$ in all the presented numerical results, which is normal, given the fact that in the latter, the impact of both turbulence and pointing errors was stronger than in the former. Furthermore, as Figure 5 demonstrates, the analytical results of our methodology are in complete agreement with the outcomes of the corresponding MC simulations. Finally, the case with $M=4$ and $N=5$, which means that more than 4-bit errors had to occur in order to consider the block as faulty, presented better performance than the case with $M=3$, while the 8-PAM format outperformed the 4-PPM, which was expected, since we considered more symbols. 


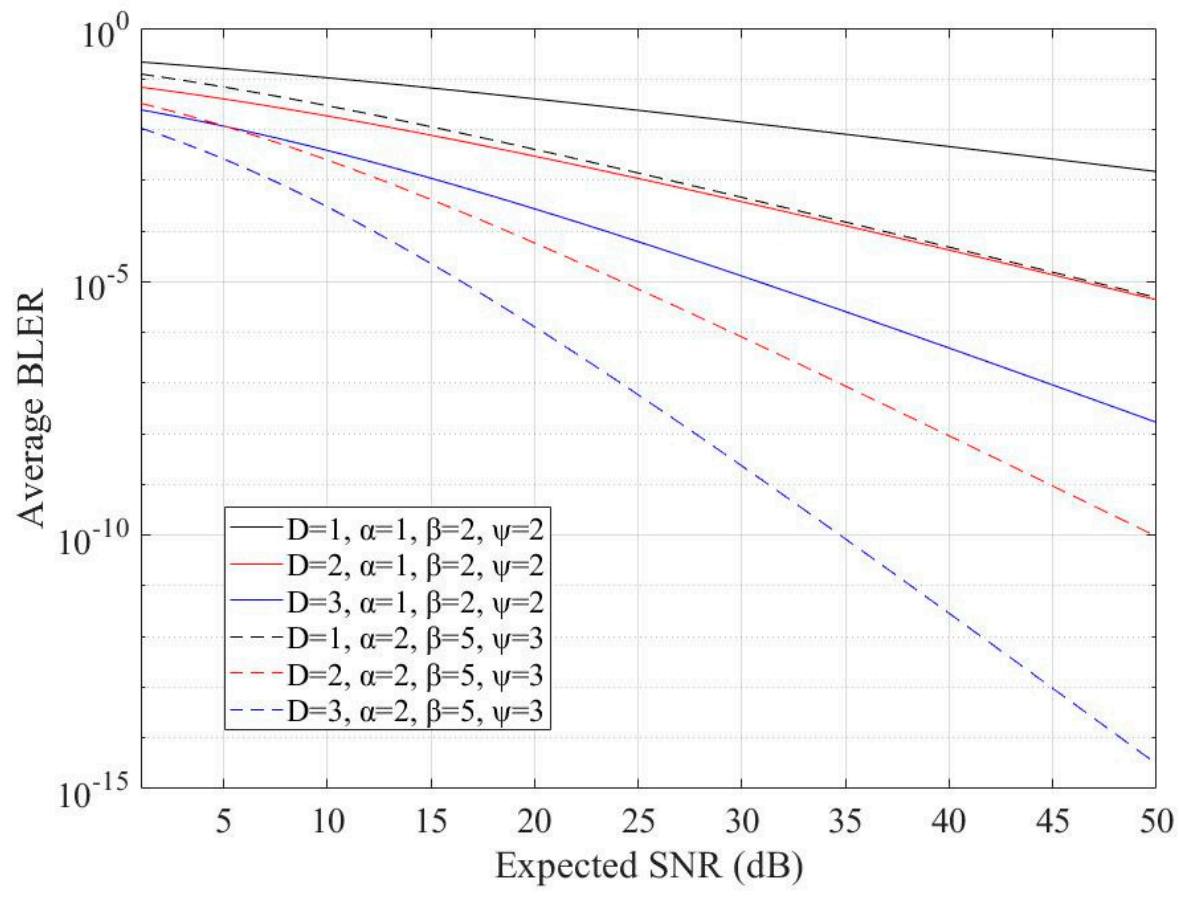

Figure 1. ABLER performance versus the expected SNR for $M=3, N=5$, and 4-PPM scheme.

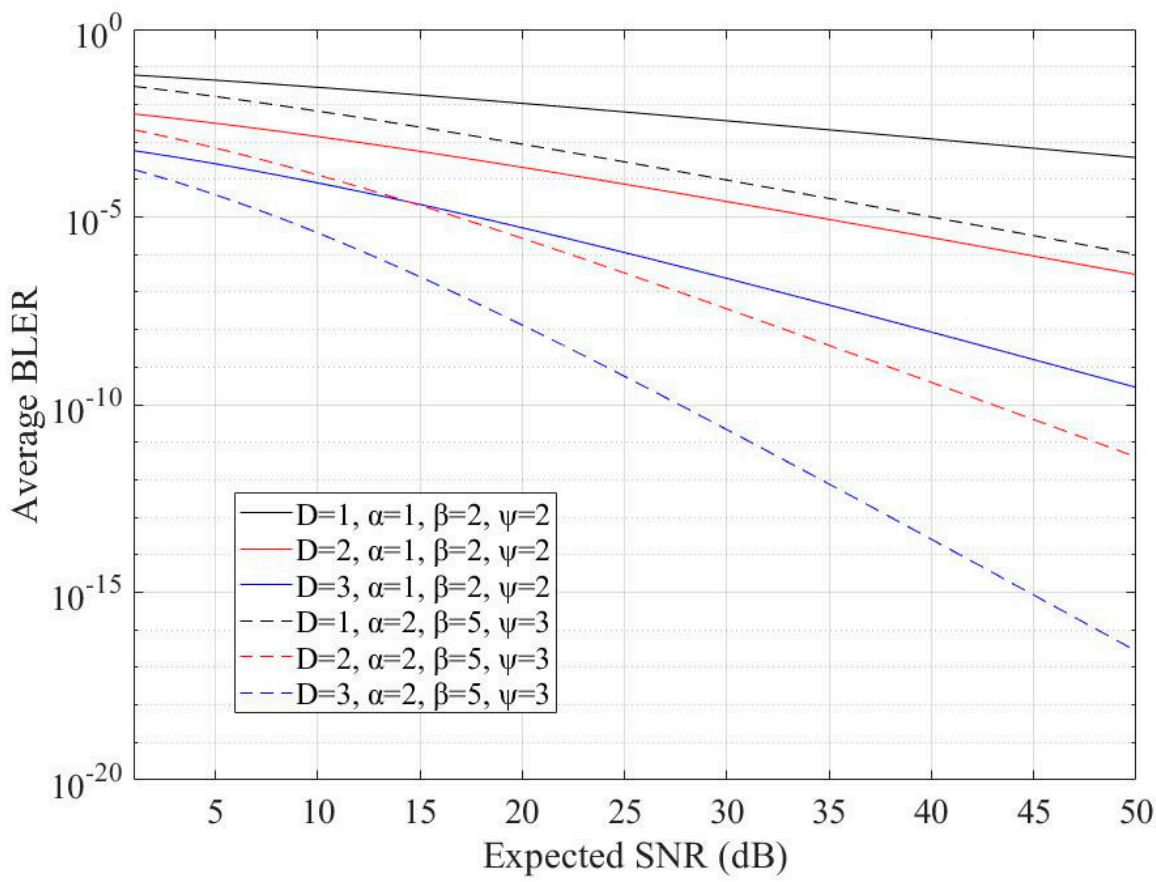

Figure 2. ABLER performance versus the expected SNR for $M=4, N=5$, and 4-PPM scheme. 


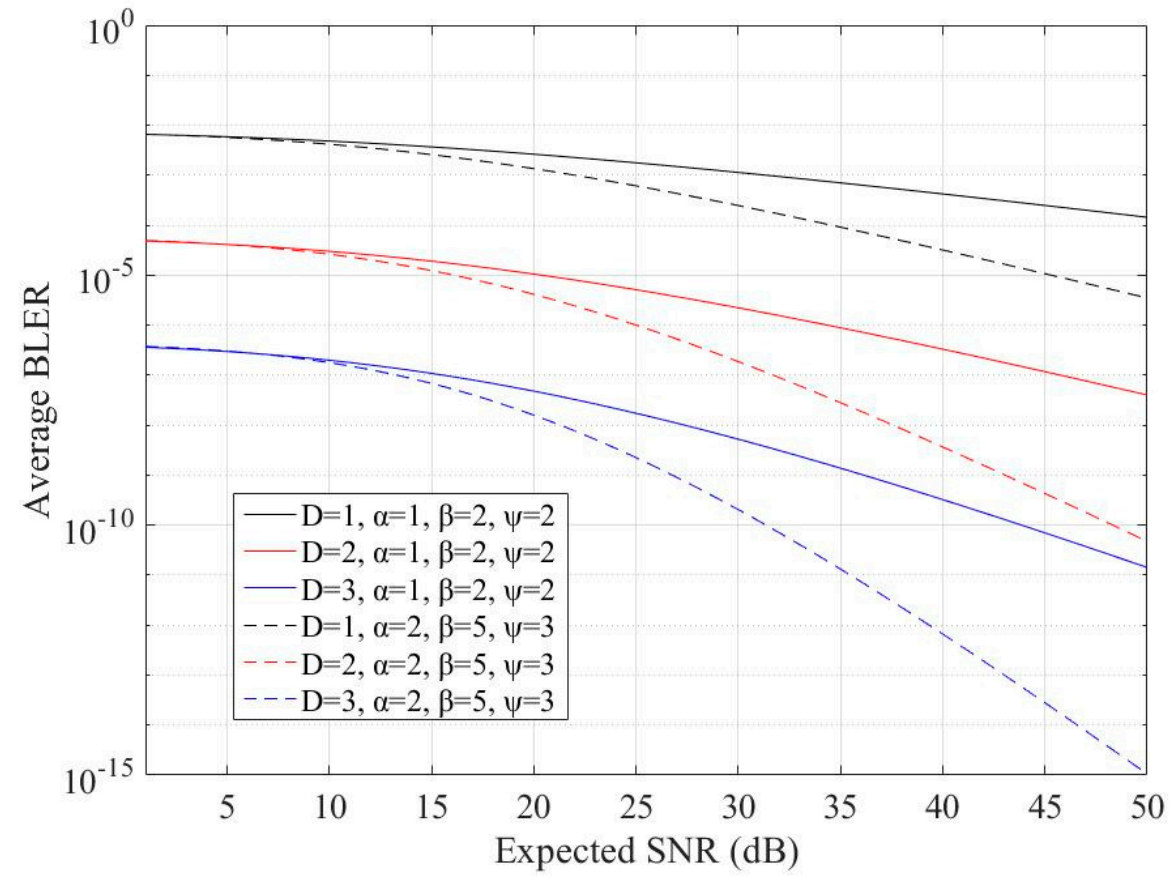

Figure 3. ABLER performance versus the expected SNR for $M=3, N=5$, and 8-PAM scheme.

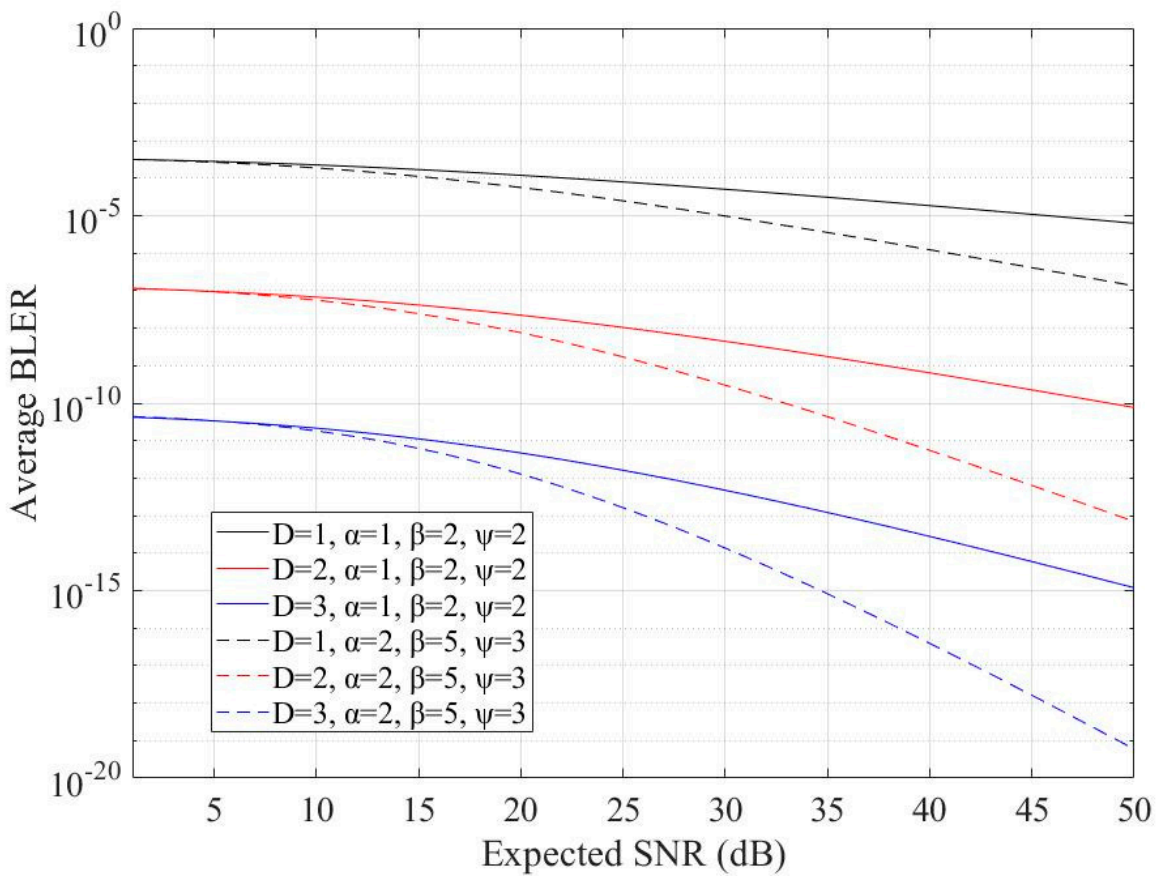

Figure 4. ABLER performance versus the expected SNR for $M=4, N=5$, and 8-PAM scheme. 


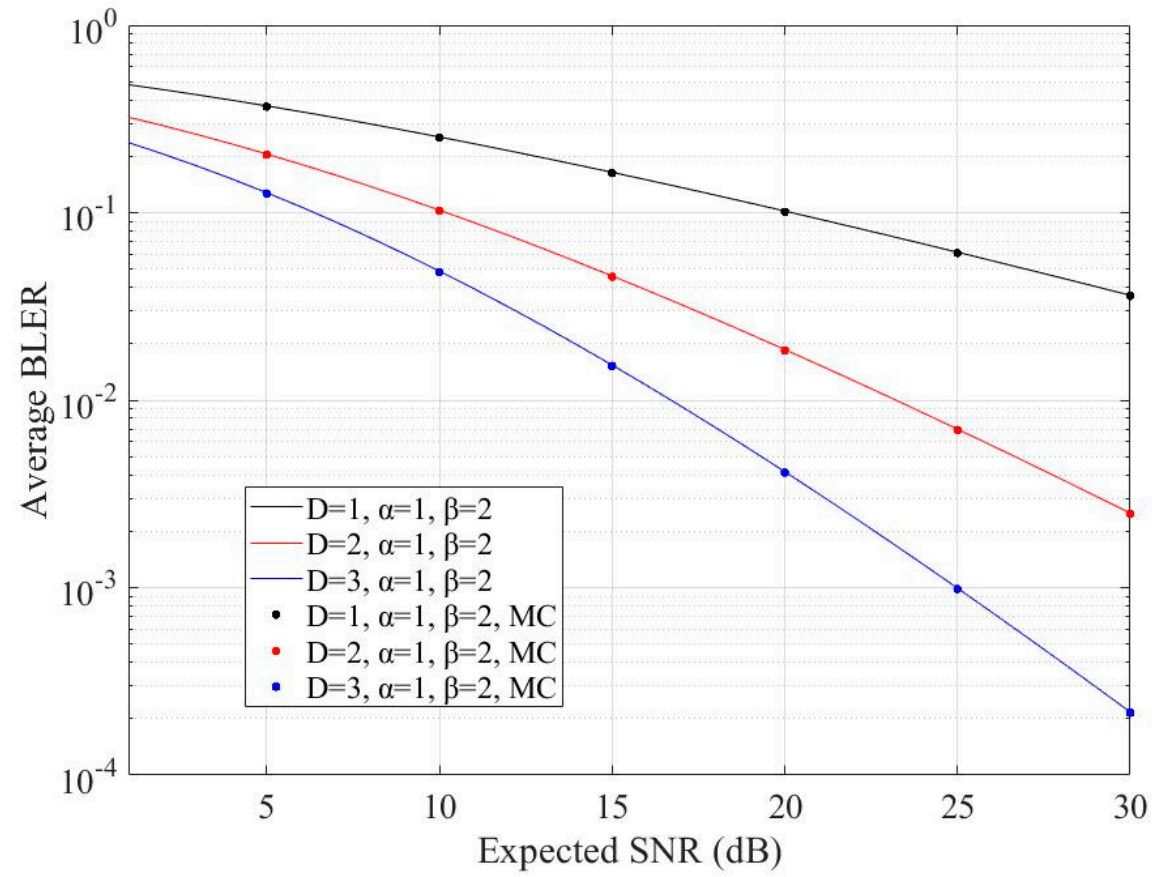

Figure 5. ABLER performance versus the expected SNR for $M=1, N=4$, negligible pointing errors impact, and 4-PPM scheme, accompanied by the corresponding Monte Carlo simulations.

\section{Conclusions}

In this work, for first time to the best of our knowledge, the average BLER performance of an FSO system with receivers' diversity which operated over the combined influence of atmospheric turbulence and spatial jitter was studied analytically. The average BLER (ABLER) is a very significant and practical metric for the design of realistic modern ultrafast FSO systems, which, so far, has not been well investigated theoretically. Novel closed-form mathematical expressions for both PPM and PAM modulation schemes were derived, and the corresponding numerical results, along with the MC simulations in some cases, for various cases of diversity, turbulence, and pointing errors strengths and erroneous bits in the block, were graphically depicted.

Author Contributions: Conceptualization, N.A.A., H.E.N., A.N.S. and G.S.T.; Formal analysis, N.A.A. and H.E.N.; Funding acquisition, H.E.N.; Investigation, N.A.A., H.E.N., E.V.C. and A.N.S.; Methodology, N.A.A., H.E.N., A.N.S. and G.S.T.; Resources, N.A.A., A.N.S. and G.S.T.; Software, N.A.A., E.V.C. and A.N.S.; Supervision, H.E.N.; Validation, N.A.A., H.E.N. and E.V.C.; Writingoriginal draft, N.A.A.; Writing-review \& editing, N.A.A., H.E.N., A.N.S. and G.S.T. All authors have read and agreed to the published version of the manuscript.

Funding: NAA acknowledges that this research was co-financed by Greece and the European Union (European Social Fund- ESF) through the Operational Program "Human Resources Development, Education and Lifelong Learning" in the context of the project "Strengthening Human Resources Research Potential via Doctorate Research" (MIS-5000432), implemented by the State Scholarships Foundation (IK $\Upsilon)$.

Conflicts of Interest: The authors declare no conflict of interest.

\section{References}

1. Androutsos, N.A.; Nistazakis, H.E.; Stassinakis, A.N.; Sandalidis, H.G.; Tombras, G.S. Performance of SIMO FSO Links over Mixture Composite Irradiance Channels. Appl. Sci. 2019, 9, 2072. [CrossRef]

2. Prabu, K.; Kumar, D.S.; Srinivas, T. Performance Analysis of FSO Links under Strong Atmospheric Turbulence Conditions Using Various Modulation Schemes. Optik 2014, 125, 5573-5581. [CrossRef]

3. Ninos, M.P.; Nistazakis, H.E.; Tombras, G.S. On the BER Performance of FSO Links with Multiple Receivers and Spatial Jitter over Gamma-Gamma or Exponential Turbulence Channels. Optik 2017, 138, 269-279. [CrossRef] 
4. Ghassemlooy, Z.; Arnon, S.; Uysal, M.; Xu, Z.; Cheng, J. Emerging Optical Wireless Communications-Advances and Challenges. IEEE J. Sel. Areas Commun. 2015, 33, 1738-1749. [CrossRef]

5. Leitgeb, E.; Lösehnigg, M.; Birnbacher, U.; Schwarz, G.; Merdonig, A. High Reliable Optical Wireless Links for the Last Mile Access. In Proceedings of the 2008 10th Anniversary International Conference on Transparent Optical Networks (ICTON 2008), Athens, Greece, 22-26 June 2008; Volume 4, pp. 178-183. [CrossRef]

6. Sandalidis, H.G.; Tsiftsis, T.A.; Karagiannidis, G.K.; Uysal, M. BER Performance of FSO Links over Strong Atmospheric Turbulence Channels with Pointing Errors. IEEE Commun. Lett. 2008, 12, 44-46. [CrossRef]

7. Khalighi, M.A.; Uysal, M. Survey on Free Space Optical Communication: A Communication Theory Perspective. IEEE Commun Surv. Tutor. 2014, 16, 2231-2258. [CrossRef]

8. Ghassemlooy, Z.; Popoola, W.O. Terrestrial Free-Space Optical Communications. In Mobile and Wireless Communications Network Layer and Circuit Level Design; InTech: London, UK, 2010. [CrossRef]

9. Djordjevic, G.; Petkovic, M.; Cvetkovic, A.; Karagiannidis, G. Mixed RF/FSO Relaying with Outdated Channel State Information. IEEE J. Sel. Areas Commun. 2015, 33, 1935-1948. [CrossRef]

10. Djordjevic, G.T.; Petkovic, M.I.; Spasic, M.; Antic, D.S. Outage Capacity of FSO Link with Pointing Errors and Link Blockage. Opt. Express 2016, 24, 219. [CrossRef] [PubMed]

11. Tsonev, D.; Sinanovic, S.; Haas, H. Complete Modeling of Nonlinear Distortion in OFDM-Based Optical Wireless Communication. J. Lightwave Technol. 2013, 31, 3064-3076. [CrossRef]

12. Dimitrov, S.; Sinanovic, S.; Haas, H. Clipping Noise in OFDM-Based Optical Wireless Communication Systems. IEEE Trans. Commun. 2012, 60, 1072-1081. [CrossRef]

13. Gappmair, W.; Hranilovic, S.; Leitgeb, E. OOK Performance for Terrestrial FSO Links in Turbulent Atmosphere with Pointing Errors Modeled by Hoyt Distributions. IEEE Commun. Lett. 2011, 15, 875-877. [CrossRef]

14. Ninos, M.P.; Nistazakis, H.E.; Sandalidis, H.G.; Stassinakis, A.N.; Tombras, G.S. Block Error Rate Performance of OOK Freespace Optical Links over Gamma-Gamma Turbulence Channels with Generalised Nonzero Boresight Pointing Errors. IET Optoelectron. 2018, 12, 269-272. [CrossRef]

15. Farid, A.A.; Hranilovic, S. Outage Capacity Optimization for Free-Space Optical Links with Pointing Errors. J. Lightwave Technol. 2007, 25, 1702-1710. [CrossRef]

16. Varotsos, G.K.; Nistazakis, H.E.; Petkovic, M.I.; Djordjevic, G.T.; Tombras, G.S. SIMO Optical Wireless Links with Nonzero Boresight Pointing Errors over M Modeled Turbulence Channels. Opt. Commun. 2017, 403, 391-400. [CrossRef]

17. Navidpour, S.; Uysal, M.; Kavehrad, M. BER Performance of Free-Space Optical Transmission with Spatial Diversity. IEEE Trans. Wirel. Commun. 2007, 6, 2813-2819. [CrossRef]

18. Tsiftsis, T.A.; Sandalidis, H.G.; Karagiannidis, G.K.; Uysal, M. Optical Wireless Links with Spatial Diversity over Strong Atmospheric Turbulence Channels. IEEE Trans. Wirel. Commun. 2009, 8, 951-957. [CrossRef]

19. Zhang, Q.; Cheng, J.; Karagiannidis, G.K. Block Error Rate of Optical Wireless Communication Systems over Atmospheric Turbulence Channels. IET Commun. 2014, 8, 616-625. [CrossRef]

20. Gappmair, W.; Hranilovic, S.; Leitgeb, E. Performance of PPM on Terrestrial FSO Links with Turbulence and Pointing Errors. IEEE Commun. Lett. 2010, 14, 468-470. [CrossRef]

21. Sandalidis, H.G.; Tsiftsis, T.A.; Karagiannidis, G.K. Optical Wireless Communications with Heterodyne Detection Over Turbulence Channels With Pointing Errors. J. Lightwave Technol. 2009, 27, 4440-4445. [CrossRef]

22. Varotsos, G.K.; Nistazakis, H.E.; Stassinakis, A.N.; Volos, C.K.; Christofilakis, V.; Tombras, G.S. Mixed Topology of DF Relayed Terrestrial Optical Wireless Links with Generalized Pointing Errors over Turbulence Channels. Technologies 2018, 6, 121. [CrossRef]

23. Al-Habash, M.A. Mathematical Model for the Irradiance Probability Density Function of a Laser Beam Propagating through Turbulent Media. Opt. Eng. 2001, 40, 1554. [CrossRef]

24. Gradshteyn, I.S.; Ryzhik, I.M. Table of Integrals, Series, and Products (Academic Press), 7th ed.; Elsevier: New York, NY, USA, 2007.

25. Uysal, M.; Li, J.; Yu, M. Error Rate Performance Analysis of Coded Free-Space Optical Links over Gamma-Gamma Atmospheric Turbulence Channels. IEEE Trans. Wirel. Commun. 2006, 5, 1229-1233. [CrossRef]

26. Bayaki, E.; Schober, R.; Mallik, R. Performance Analysis of MIMO Free-Space Optical Systems in Gamma-Gamma Fading. IEEE Trans. Commun. 2009, 57, 3415-3424. [CrossRef]

27. Ismail, T.; Leitgeb, E.; Ghassemlooy, Z.; Al-Nahhal, M. Performance Improvement of FSO System Using Multi-pulse Pulse Position Modulation and SIMO under Atmospheric Turbulence Conditions and with Pointing Errors. IET Netw. 2018, 7, 165-172. [CrossRef]

28. Jurado-Navas, A.; Garrido-Balsells, J.M.; Paris, J.F.; Castillo-Vázquez, M.; Puerta-Notario, A. Impact of Pointing Errors on the Performance of Generalized Atmospheric Optical Channels. Opt. Express 2012, 20, 12550-12562. [CrossRef]

29. Adamchik, V.S.; Marichev, O.I. The Algorithm for Calculating Integrals of Hypergeometric Type Functions and Its Realization in REDUCE System. In Proceedings of the International Symposium on Symbolic and Algebraic Computation, Tokyo, Japan, 20-24 August 1990; pp. 212-224. [CrossRef]

30. Odeyemi, K.O.; Owolawi, P.A.; Srivastava, V.M. Performance Analysis of Block Error Rate for SIM-FSO System with Spatial Diversity over Gamma-Gamma Fading and Pointing Error Channel. In Proceedings of the 2017 IEEE AFRICON, Cape Town, South Africa, 18-20 September 2017; pp. 115-120. [CrossRef] 
31. Loskot, P.; Beaulieu, N.C. Prony and Polynomial Approximations for Evaluation of the Average Probability of Error over Slow-Fading Channels. IEEE Trans. Veh. Technol. 2009, 58, 1269-1280. [CrossRef]

32. The Wolfram Functions Site. Available online: http:/ / functions.wolfarm.com (accessed on 10 May 2021).

33. Sandalidis, H.G.; Chatzidiamantis, N.D.; Karagiannidis, G.K. A Tractable Model for Turbulence- and Misalignment-Induced Fading in Optical Wireless Systems. IEEE Commun. Lett. 2016, 20, 1904-1907. [CrossRef] 